Aus dem Zoologischen Institut München.

\title{
Über den Aufbau der Speicheldrüsenkerne der Chironomuslarve.
}

Von

Dr. Hubert Erhard.

Hierzu Tafel V und eine Textfigur.

\section{Einleitung.}

Es gibt verhältnismässig wenig Metazoen, welche in bestimmten Organen eine ganz bezeichnende, immer wiederkehrende Form des Zellkernaufbaues zeigen, die einzig und allein der betreffenden Tierart oder dem betreffenden Organ zukommt. Mit anderen Worten, die verschiedensten Organe der verschiedensten Tierarten haben oft die grösste Ähnlichkeit im Aufbau ihrer Zellkerne. Eine Ausnahme von dieser Regel findet sich vielfach bei Arthropoden vor. Wir müssen hier zwischen zweierlei ganz bezeichnenden Typen unterscheiden, zwischen den in Anpassung an eine bestimmte lebhafte Zelltätigkeit umgeformten Kernen und solchen, die nur wäbrend der Entwicklungsperiode des Tieres charakteristisch geformt sind, um später sich mehr dem allgemeinen Kernschema zu nähern. Wir wollen die einen der Einfachheit wegen kurz die Funktions-, die anderen die Entwicklungskerne nennen. Als Beispiel der ersteren mögen die von Meves besonders schön dargestellten Spinndrüsenkerne gelten, die sich wohl in Anpassung an die ausserordentlich hohe Funktion ihrer Zellen stets in der merkwürdigsten Weise umgeformt haben. Unter den Entwicklungskernen bat seit der bekannten Untersuchung Balbianis (1) der Typus, den dieser Forscher in den Speicheldrüsen der Chironomuslarve aufgefunden hatte, stets besondere Aufmerksamkeit gefunden. Im Kern befindet sich ein langer, gewundener, wahrscheinlich aus einzelnen aneinander gereihten Scheiben zusammengesetzter Faden, dessen beide Enden sich träubchenförmig erweitern. In der Nähe dieser Erweiterungen befindet sich je ein, den Faden 
umgebender Ring. Balbiani (1) hat seine, mit den damaligen Hilfsmitteln wohl nicht zu übertreffende Untersuchung am lebenden Objekt gemacht; er konnte also sicher gehen, keine Kunstprodukte vor Augen zu haben. Die Deutung der verschiedenen Bestandteile schien ihm ohne weiteres klar: die erweiterten Enden der Făden, sagte er, sind die Nukleolen, die Fäden selbst stellen das Chromatin des Kernes dar. Gerade damals batte man ja begonnen, die indirekte Kernteilung näher zu studieren, nichts lag also naher, als den Faden des Chironomus-Speicheldrüsenkernes mit dem sich zur Chromosomenbildung fädig umgeformten Chromatin der sich teilenden Zelle zu vergleichen. Wilson (15) hat in diesem Sinne diesen Kernen den Namen "Spiremkerne" verlieben, und dass die
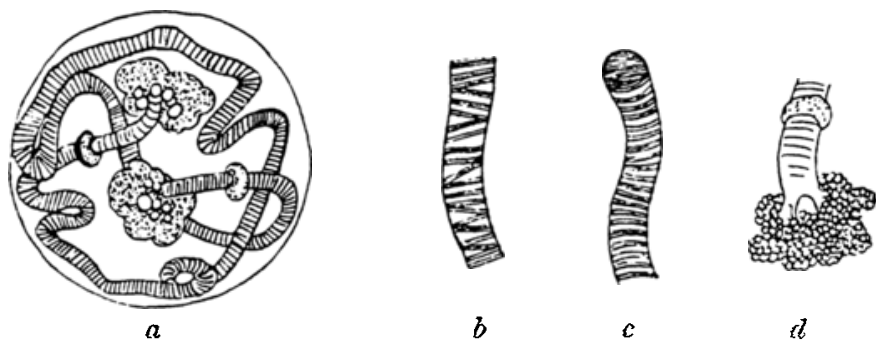

Fig. A. Kern einer Speicheldrüsenzelle der Chiro n o mus larve nach Balbiani. a = Nukleolen, Kugeln unterbalb der Nukieolen, Kemfaden und Kernringe sichtbar. b und $c=$ Rernfadenstuxcie bei stärkerer Vergrösserung. $d=$ traubenförmiger Nakleolus.

Balbianische Deutung die allein herrschende blieb, erkennt man besonders aus unseren zusammenfassenden Werken über die Zelle, so ausser dem von Wilson selbst, den Werken von Henneguy $(7,8)$, O. Hertwig (9), Carnoy (3), Gurwitsch (5) und Heidenbain (6).

Es bat sich nach der Untersuchung Balbianis in der Folgezeit wohl kein Objekt gefunden, das so schön die Struktur erkennen lässt, die den Speicheldrüsenkern von Chironom us auszeichnet, immerhin finden wir manche Berichte von ähnlichen Vorkommnissen auch bei anderen Arthropoden von Henneguy (7, 8). Am Darmepithel der Larve von Ptychoptera fand Van Gebuchten (7) gleichfalls solche Gebilde und Strasburger (14) zeigte, dass sie auch in der Botanik vorkommen. 
Endlich berichtet 0 . vom $R$ ath von einer polyzentrischen Anordnung des Chromatins bei Kernen, die ihm bei Schnitten durch die Kopfregion von Anil o cra mediterranea zu Gesicht kamen. Diese lassst sich vielleicht auch, wie ich glaube, mit unserer eben besprochenen Kernform vergleichen. Herr Geheimrat von Hertwig hatte die Güte, mich auf Práparate unserer Institutssammlung aufmerksam zu machen, die er selbst vor etwa 25 Jahren gefertigt hatte, und die nicht nur an Chironomus die Angaben Balbianis bestätigten, sondern auch an den Speicheldrüsen der Larven von $\mathrm{Culex}$ pipiens, allerdings in weit zarterem Aufbau, solche "Spiremkerne" erkennen liessen.

\section{Material und Methoden.}

Für die folgende Untersuchung wurden vor allem die Speicheldrüsen der Chir on om us larve verwendet. Diese Larven befanden sich kurz vor der weiteren Metamorphose. Im Gegensatz zu Henneguy (7), der die Drüsen so herauspräparierte, dass er den Kopf der Tiere abriss, wobei die Speicheldrüsen mitgingen, die dann vom Kopf losgelöst wurden, schnitt ich nach dem ersten, dem Kopf folgenden Segment durch. Entweder quollen nun die Speicheldrüsen ganz von selbst aus dem Körper hervor und es konnte ihr feiner Ausführgang, um sie loszulösen, durchschnitten werden, oder ein leichter Druck auf die folgenden Segmente liess sie hervortreten. Die abgelösten Speicheldrüsen: wurden entweder mit Sublimat-Eisessig (Subl. 1/2 conc. +2 Teile Eisessig) oder in Fle m m in g schem Gemisch fixiert. Eine wesentlich bessere Konservierung ergab die erstere Art. Die in Sublimat fixierten Objekte wurden als Ganzes in Boraxkarmin gefarbt. Ein Teil von ihnen wurde zu Totalprăparaten benutzt. Bemerkenswert ist, dass kurzes Färben, etwa 1/2 Stunde lang, mit darauffolgendem 8-10 tăgigem Ausziehen die besten Präparate lieferte. Bei den für Schnittpräparate bestimmten Objekten, die $3-20 \mu$ dick geschnitten wurden, wurde vorerst nicht extrahiert. Gefärbt. wurde dann ausser mit Safranin-Lichtgrün und dem Gemisch von Ehrlich-Biondi-R. Heidenhain, wobei jedesmal zuerst Boraxkarmin entfernt wurde, vor allem nach der sogenannten Obst schen Nukleolenfarbung. Die nicht extrahierten Boraxkarminschnitte kamen auf $11^{1 / 2}-4$ Stunden in eine sehr verdünnte wässerige 
Methylgrünlösung. War die Lösung so konzentriert, dass noch deutlich ihr grüner Farbton zu erkennen war, so kam schon eine Überfübung zustande. Ich tat in den darauffolgenden $70 \%$ Alkohol in diesem Fall einige Tropfen $5 \%$ iger ammoniakalischer Lösung, die in etwa einer Minute den überschüssigen Farbton auszog. Die besten Bilder bekam ich aber, wenn ich die Methylgrünlösung so sehr verdünnte, dass sie mehr einem ein wenig blaugrün schillernden Wasser glich und darin die Schnitte $1^{1} / 2$ Stunden, also weit geringere Zeit als Obst (12), der 2-3 Stunden fürbte, liess. Die in Flemmings Gemisch fixierten Präparate kann ich übergehen, da sie mich nach lieiner Fürbung befriedigten. Es ist ferner natürlich, dass den Untersuchungen am gefarbten Objekt die am lebenden vorangingen. Ausser den von Balbiani (1) angegebenen Methoden wurden noch Pressversuche unternommen. Ferner wurden noch Prïparate vom Darm der Larven ebenso fixiert und gefürbt. Ausser diesen eigenen Priparaten standen mir zur Untersuchung noch die von Herrn Geheimrat von Hertwig gefertigten zur Verfügung, die ausser den Chironomus- auch die Culex pipiens-Speicheldrüsen betrafen. Bei Chironomus handelte es sich hier manchmal wohl um noch jüngere Tiere. In beiden Fallen war mit Chromsiture fixiert und mit Safranin total gefärbt worden. Ich möchte auch an dieser Stelle Herrn Geheimrat von Hertwig für die Überlassung der Präparate meinen verbindlichsten Dank sagen.

\section{Eigene Beobachtungen.}

Die eigenen Untersuchungen begannen mit dem Studium des lebenden Materials im Blut des Tieres. Ich kann mir die Beschreibung der Ergebnisse dessen ersparen, da sie nur das bestätigen, was schon Balbiani (1) erkannt hatte (vergl. Textfigur A). Nur über einen Punkt, über den sich dieser Forscher nicht völlig klar wurde, möchte ich berichten. Balbiani lionnte nämlich nicht mit Sicherheit entscheiden, ob der Faden sich aus einzeinen aneinander gereihten Scheiben zusammensetzt, wenngleich er diese Art des Aufbaues für den wahrscheinlichsten hält. Ich versuchte, den Zusammenhalt des Gebildes durch Druck mit dem Deckgläschen zu lockern, was auch schliesslich gelang. Der Faden zerfiel tatsächlich in einzelne Scheiben. 
Diese Behauptung möchte ich aufrecht erhalten, auch nachdem ich nachträglich durch eine eben erschienene Arbeit $\mathrm{Her}$ werdens (10) auf eine andere Deutung des Aufbaues des Kernfadens aufmerksam gemacht wurde. Nachdem schon früher Korschelt (11) angegeben hatte, dass seine Zusammensetzung aus aneinandergereihten Scheiben nur eine scheinbare sei, in Wirklichkeit vielmehr, wie sich durch Druck feststellen lasse, der Kernfaden aus einer aufgerollten Spirale bestehe, schliesst sich Herwerden auf Grund eigener Untersuchungen dieser Deutung vollauf an. Er glaubt "unwiderleglich nachzuweisen", dass beim Kernfaden nein spiral förmig gewundener Faden vorliegt, dessen Windungen .... eine achromatische Substanz umlagern". Anschliessend an eine Angabe von Bonnevie (2), die beim Furchungskern von Ascaris die Chromosomen in der Telophase als gewundene Spiralfäden beschreibt, sagt er: "Nach meiner Auffassung muss also die Struktur dieser Kerne" (sc. Chironomus!) „nicht als eine isoliert dastehende betrachtet werden, sondern als eine sehr typische Strulitur, zu deren zeitlicher oder dauerhafter Umgestaltung vermutlich jedem tierischen und pflanzlichen Kern die Fahigkeit inne wohnt". Aber die Bestandteile der Chironomuskerne weichen doch, wie wir sehen werden, wesentlich von den Chromosomen ab. Herverden konnte ferner auch nicht die Identität der Chironomuskernfäden mit Chromosomen beweisen, da er, wie er selbst angibt, nie eine Zellteilung in den Speicheldrüsen beobachten konnte. Ferner sind die Angaben von spiralig gedrehten Chromosomen doch relativ selten - Heidenhain (6, pag. 174-176) z. B. glaubt, dass die Chromosomen der Spireme der Salamanderkiemenblättchen eine Drehung und Gegendrehung zeigen -, so dass ich dieser Verallgemeinerung $\mathrm{Herwerdens}$ auch in bezug auf die Chromosomen skeptisch gegenüberstehe.

Sehr überrascht war ich, als ich anfänglich am gefärbten Objekt die einzelnen, das Kerngerüst zusammensetzenden Teile manchmal nicht so gut wiedererkannte, wie bei den Untersuchungen im lebenden Zustand. Ganz besonders gilt dies von den Totalpräparaten. Man kann offen sagen, dass sich kaum ein anderes histologisches Objekt so sehr zur Lebend-Beobachtung eignet, wie die Kerne der $\mathrm{Ch}$ ir o n o mus speicheldrüsen, und dass es überhaupt wundernehmen muss, wie bei Objekten, die so leicht 
zu beschaffen sind, die Histologie verhältnismăssig selten Studien von Zellen in ihrem Naturzustand ausführt. Nur einige Einzelheiten des Kernaufbaues gelang es am gefärbten Material zu klären. For allem zeigten die ganz kleinen, also ganz jungen Larven angehörigen Kerne einen einfachen Nukleolus, von dem aus die beiden Schleifenenden entsprangen. Erst bei älteren Larven, die kurz vor der weiteren Metamorphose sich befanden, waren stets zwei Nukleolen anzutreffen, endigte der aufgerollte Faden also frei, während er im ersteren Fall in sich selbst zurücklief. Es ist also erklärlich, warum Balbiani (1) einmal ein einfaches Kernkörperchen und einmal zwei Nukleolen antraf. Im übrigen ist der Nukleolus ganz so aufgebant, wie es Balbiani angibt. Ein aus dem Kernfaden hervorgehender Stiel verïstelt sich mehrfach, und an den Enden dieser Verzweigungen sitzen wie Traubenbeeren kleine dicht gedrängte Kügelchen. Unsere Fig. 2 zeigt einen quer getroffenen, rot gefärbten Nukleolus. Der rote Kreis in der Mitte stellt den Querschnitt des Mittelstiels vor, dieser wird auf drei Seiten (oben, rechts und rechts unten) von den hier quer getroffenen roten Beeren umgeben. Diese scheinen längsovale Form zu haben und in zwei Lagen, einer äusseren und einer inneren, angeordnet zu sein. Die Oberfluche des Nukleolus ist keine verhältnismässig gleichartige Flache, vielmehr springt jeweils ein grösserer oder kleinerer Komplex von Beeren aus dem kugelförmigen Gebilde wieder zusammen kugelförmig hervor, so dass der Anblick des Ganzen einer aus einzelnen Kugeln zusammengekneteten Kugel gleicht. Dies erkennt man bei Betrachtung von Fig. 1, die den ganzen Nukleolus in dunkelkirschroter Farbe zeigt. Den Stiel umgeben an del Stelle, an der er sich verzweigt, grössere Kügelchen, die schon Balbi a n i (1) aufgefunden hatte. In unserer Fig. 2 sind es die blaugrün gefärbten Kugeln, die um den Stiel gelagert sind. Ibre Zabl ist nach meiner Schätzung etwa zehn.

Der eigentliche Faden setzt sich, wie oben schon angegeben, aus einzelnen Scheiben zusammen. Im fixierten Zustand erweist er sich ziemlich brüchig, was daraus erhellt, dass er durch das Messer oft wohl in seiner Lage verändert und eher durchgerissen als durchschnitten wird. Nach Heidenhain (6) sind ja überhaupt oft Kernstrukturen recht brüchig. Gleich in der Nähe des Nukleolus wird der Kernfaden von zwei Ringen umgeben (Fig. 1 
und 2). Balbiani (1) fand einen einzigen solchen Ring auf. Jüngere Larven besitzen in der Tat, wie ich feststellen konnte, nur einen, ältere dagegen haben zwei Ringe. Balbiani konnte die feinere Zusammensetzung des Ringes nicht ermitteln. An gefärbten Schnittpräparaten erwiesen sie sich mir als halbrund erhaben, zusammengesetzt aus dicht aneinander gelagerten Kügelchen (Fig. 2), ähulich wie der Nukleolus. In der Breitseite des Ringes folgen sich wahrscheinlich drei Reihen von Kügelchen. Die Kugeln sind aber nicht wie die der Kernkörperchen längsoval, sondern kreisrund. Dies liess sich an einem Schnitt feststellen, der eben einen solchen Ring traf und ihn durchriss, so dass die Kügelchen im Kern zerstreut herumlagen. Über den weiteren Verlauf des Kernfadens ist folgendes zu sagen: Balbiani (1) fand, dass er sich zuweilen spaltet, eine Wahrnehmung, die ich nie machen konnte. Immer verlief er - an gefärbten Totalpräparaten wie am ungefärbten ganzen Objekt liess sich dies mit aller Sicherheit feststellen - einheitlich bis zum anderen Nukleolus. So waren die Verhaltnisse wenigstens an den Speicheldrüsenkernen der Chironomuslarve. $O b$ dasselbe für die von $\mathrm{Culex}$ pipiens zutrifft, wage ich nicht $z u$ entscheiden. Hier ist der ganze Faden ungleich feiner und lünger, so dass das ganze Bild viel schwerer zu deuten ist. Auch der Darm der Chironomuslarve besitzt, wie ich feststellen konnte, solche Spiremkerne. Hier konnte gleichfalls nicht mit Bestimmtbeit nach einer Richtung bin entschieden werden. Alle Scheiben des Fadens erweisen sich als völlig gleichartig. O. Hertwig (9) berichtet zwar, dass der Kernfaden der Speicheldrüse der Chironomuslarve „im gefürbten Prïparate eine regelmässige Aufeinanderfolge tingierter und nicht tingierter Scheiben erkennen lässt"; bei meinen Versuchen, sowohl mit einfachen wie mit Doppelfärbungen, färbten sich die Scheiben jedesmal völlig gleichmässig. Ich glaube, dass die gegenteilige Auffassung daher rührt, dass die freien Zwischenräume zwischen je zwei gefärbten Scheiben einen Wechsel von gefärbten und ungefärbten vortauschen. Zwischen je zwei Scheibenrändern besteht nämlich eine Lücke. Wie die Scheiben eigentlich miteinander verbunden sind, ob sie im Zentrum ihres Radius vielleicht zusammenhängen, konnte nicht entschieden werden.

Wichtiger als diese doch nur mehr äusserlichen Feststellungen sind, glaube ich, die Erkenntnisse, die über die Bedeutung des 
Kernapparates gewonnen wurden. Ich muss hier weiter ausholen: Bekanntlich hat Obst (12) gelegentlich seiner Studien über Molluskeneier eine Methode entdeckt, mit der es möglich ist, das Chromatin des Kernes und die Nukleolarsubstanz im engeren Sinne verschieden zu färben. Boraxkarmin färbt das Chromatin, Methylgrün die Nukleolarsubstanz. Zur Unterscheidung von Basiund Oxychromatin ist ferner namentlich von Heidenhain (6) mit Erfolg das Ehrlich-Biondi-Heidenhainsche Gemisch verwandt worden. Ich muss mich hier mit dieser Andeutung begnügen, da Heidenhain in wohl erschöpfender Weise die Theorie der Kernfürbung behandelt hat und verweise deshalb für alles Weitere auf sein Werk. Beim Lesen dieser Schriften dachte ich an die Chironomusspeicheldrüsenkerne, deren eigentümliche Strulstur, ferner ihr Entwicklungscharakter, wie ich glaubte, ein Licht auf die Zusammensetzung des Kernes und auf die Bedeutung der Nukieolen werfen lsönnten. Ein grosser Teil dieser Hoffhungen wurde zu nichte, da merkwürdigerweise die EhrlichBiondi-Heidenhainsche Färbung nie recht gelang, und ich nur Material von ziemlich grossen Larven bekam, der Hauptreiz aber wohl in der Erforschung der Entstehung des eigenartigen Kernaufbaues bestanden hätte. So konnte demn nur die Zusammensetzung des Kernes in einem einzigen Entwicklungsstadium näher studiert werden. Hatte B a lbian i (1) das erweiterte Ende der Fäden als Nukleolen gedeutet, so blieb immer noch die Natur der Ringe unbekannt, und des weiteren fiel mir an den Prïparaten von Herrn Geheimrat von Hertwig auf, dass sich einmal der Faden lebhafter mit Safranin, einmal der Nukleolus intensiver mit diesem Farbstoff fărbte, d. h. dass eine wechselvolle Neigung in der Aufnahme basischer Anilinfarbstoffe besteht. Die Tiere, an denen dies stattfand, mussten jünger gewesen sein als die mir zur Verfügung stehenden. An diesen letzteren war stets eine ganz bestimmte Neigung, Farbstoffe aufzunehmen, festgelegt, und zwar genau die entgegengesetzte, die man erwartet hätte. Figur 1 stellt uns einen Teil eines Kernes dar, der stark mit Methylgrün überfarbt ist. Als echtes Chromatin, d. b. mit Boraxkarmin gefärbt, ist vor allem der "NNukleolus" zu erkemnen. Eine annliche Reaktion zeigen noch die beiden Ringe. Die rote Farbe besitzt in der Zelle ausserdem nur noch das Sekret. Also trotz der absichtlich erzielten Methylgrïnüber- 
färbung, die bei starker Verdünnung dieser Flüssigkeit etwa drei Stunden währte, keinerlei Tinktion des "Nukleolus" mit dieser Nukleolenfärbung.

Nun las ich freilich erst nach Abschluss dieser Untersuchung, dass schon Korschelt (11) die gleiche Fürbung anwandte und auf Grund derselben von einem Unterschied in der Färbung von Kernfaden und Nukleolus berichtet, von denen sich ersterer mit Methylgrün, letzterer mit Boraxkarmin tingieren soll. Wir wissen jedoch nicht, in welcher Konzentration $\mathrm{K}$ orschelt Methylgrün anwandte. Da wir aber erst seit der bekannten Obstschen $\mathrm{Ab}$ handlung (12) zu der Auffassung gelangt sind, dass M e thylgrün in äusserster Verdünnung a uf Boraxkarmin-Praparate angewandt eine elektive Färbung der echten Nukleolarsubstanz sei, so glaube ich, ist trotzdem mein Versuch durch die Untersuchung Korschelts nicht ganz ïberflüssig gemacht. Dazu kommt, dass Korschelt die Ringe mit Methylgrün, ich jedoch mit Boraxkarmin gefärbt vorfand.

Fig. 2 gibt ein Präparat wieder, das nur $1 \frac{1}{2}$ Stunden sich in einer Methylgrünlösung befand, die so sehr verdünnt war, dass das Wasser kaum noch einen blaugrünen Schimmer zeigte. Wir sehen hier nur den Kernfaden von der Nukleolenfärbung betroffen, alles übrige ist durch Boraxkarmin tingiert. Heller leuchtet das Sekret aus dem kompakten, tief dunkelroten Plasma hervor, ') von brennendstem Rot strahlen der "Nukleolus" und die beiden Ringe, sowie der Stiel des sogenannten Kernkörperchens. Aber noch eines fällt uns an den beiden Figuren auf, was im lebenden Zustand an den Kernen nicht zu beobachten war und auch am gefärbten Objekt bisher von den Autoren übersehen wurde. Den vom bisher beschriebenen Kernapparat frei gelassenen Teil des Kernes nimmt ein Maschenwerk dicht aneinandergereihter Chromatinkügelchen ein. Die Anordnung derselben ähnelt lebhaft der, die Heidenhain (6, pag. 153) mit Vanadiumhämatoxylin für die Chromiolen der Kerne der Keimlager des Salamanderdarmepithels zur Darstellung bringen konnte.

Es kann nach alledem kein Zweifel sein, dass in den Kernen der Chironomusspeicheldrüsen, wenigstens auf einem gewissen Entwicklungsstadium, der Kernfaden die

1) In der Reproduktion wurde die kirschrote Farbe des Plasmas und die gelbrote des Sekrets durch gewöhnliches Rot ersetzt. 
Nukleolarsubstanz darstellt, wahrend die echten chromatischen Elemente in den sogen. Nukleolen, den Ringen und endlich dem Maschenwerk der Kernchromiolen festgelegt sind. Nicht immer scheint dieses Verhältnis zu bestehen, wie wir aus der oben angegebenen Verschiedenheit der Neigung, Safranin aufzunehmen, gesehen haben. In ähnlicher Weise, wie dies $\mathrm{Obst}(12)$ an Molluskeneiern beobachtete, mag ein Wechsel im Chemismus des Kernapparates, der bier auch mit einem Wechsel seiner Struktur verbunden ist, mit dem der allmählichen Entwicklung des Organismus Hand in Hand gehen oder es mögen verschiedene Funktionszustănde der Sekretion bestimmend auf den chemischen Zustand des Kernapparates einwirken. Experimentelle Untersuchungen an den verschiedensten Entwicklungsstadien könnten auf diese wichtige Frage Licht werfen. Ich bedauere, dass ich aus dem ausseren Grunde, weil mir solches Material nicht zur Verfügung stand, dieser Aufgabe nicht näher treten konnte.

München, April 1910.

\section{Literaturverzeichnis.}

1. Balbiani, E. G.: Sur la structure du noyean des cellules salivaires chez les larves de Chironomus. Zool. Anz., 4. Jahrg., 1881.

2. Bonnevie, Kristine: Chromosomenstudien I. Arch. f. Zellforsch., Bd. 1, 1908.

3. Carnoy, J. B.: La biologie cellulaire. Lierre 1884.

4. Gehuchten, Van: L'axe organique du noyeau. La cellule. T. 5. (Zitiert nach $\mathrm{Heidenhain.)}$

5. Gurwitsch, Alex: Morphologie und Biologie der Zelle. Jena 1904.

6. Heiden hain, Mart.: Plasma und Zelle. 1. Abt., 1. Lief., Jena 1907.

7. Henneguy, L. F.: Leçons sur la cellule. Paris 1896.

8. Derselbe: Les Insectes. Paris 1904.

9. Hertwig, O.: Allgemeine Biologie, 2. Aufl. Jena 1906.

10. Herwerden, M. A., van: Über die Kernstruktur in den Speicheldrüsen der Chironomuslarve. Anat. Anz., Bd. 36, 1910.

11. KorscheIt, Eugen: Über die eigentümlichen Bildungen in den Zellkernen der Speicheldrüsen von Chironomus plumosus. Zool. Anz., Bd. 7, 1884. 
12. Obst, Paul: Untersuchungen über das Verhalten der Nukleolen bei der Eibildung einiger Mollusken und Arachnoïdeen. Zeitschr. f. wiss. Zool., Bd. 66, 1899.

13. Rath, 0 . vom: Über eine eigenartige polyzentrische Anordnung des Chromatins. Zool. Anz., 13. Jahrg., 1890.

14. Strasburger, Ed.: Das botanische Praktikum. 2. Aufl., Jena 1887. (Zitiert nach 0 . Hertwig.)

1.. Wilson, E. B.: The Cell in Development and Inheritance. Jew York 1896.

\section{Erklärung der Abbildungen auf Tafel V.}

Die beiden Figuren sind mit dem Zeichenapparat bei Zeiss Kompens.-Ok. 8 und homog. Immers. $2 \mathrm{~mm}$ auf Objekttischhöhe entworfen. Die Vergrösserung ist also eine 1000 fache.

Fig. 1. Chironomuslarve, Stück einer Speicheldrüsenzelle. Im grünen Plasma rot gefärbtes Sekret. Der teilweise getroffene Kern zcigt den "Nukleolus" (dunkelkirschrot), die Ringe und Chromiolen und den Kernfaden (grün). Fixierung: Subl.-Eisessig. Fürbung: Boraxliarmin, Methylgrün. Methylgrünüberfïirbung. $10 \mu$.

Fig. 2. Gleiches Objekt. Plasma mit Sekret. (Um Kosten zu sparen wurde das kirschrote Plasma und das gelbrote Seliret in einheitlichem Farbton wiedergegeben.) Der Kern ist teilweise getroffen. Der Schnitt geht gerade quer durch die Mitte des "Nukleolus" in der Föhe seines Stiels und der diesen umgebenden grösseren Kügelchen hindurch. "Nukleolus" rot, Kügelchen blaugrün, Stiel rot. Der folgende Kernfaden (blaugrün) mit den Ringen (rot) liegt etwas tiefer. Chromiolen rot. Fixierung: Subl.-Eisessig. Färbung: Boraxlrarmin und sehr verdünnte Methylgrünlösung. $20 \mu$. 


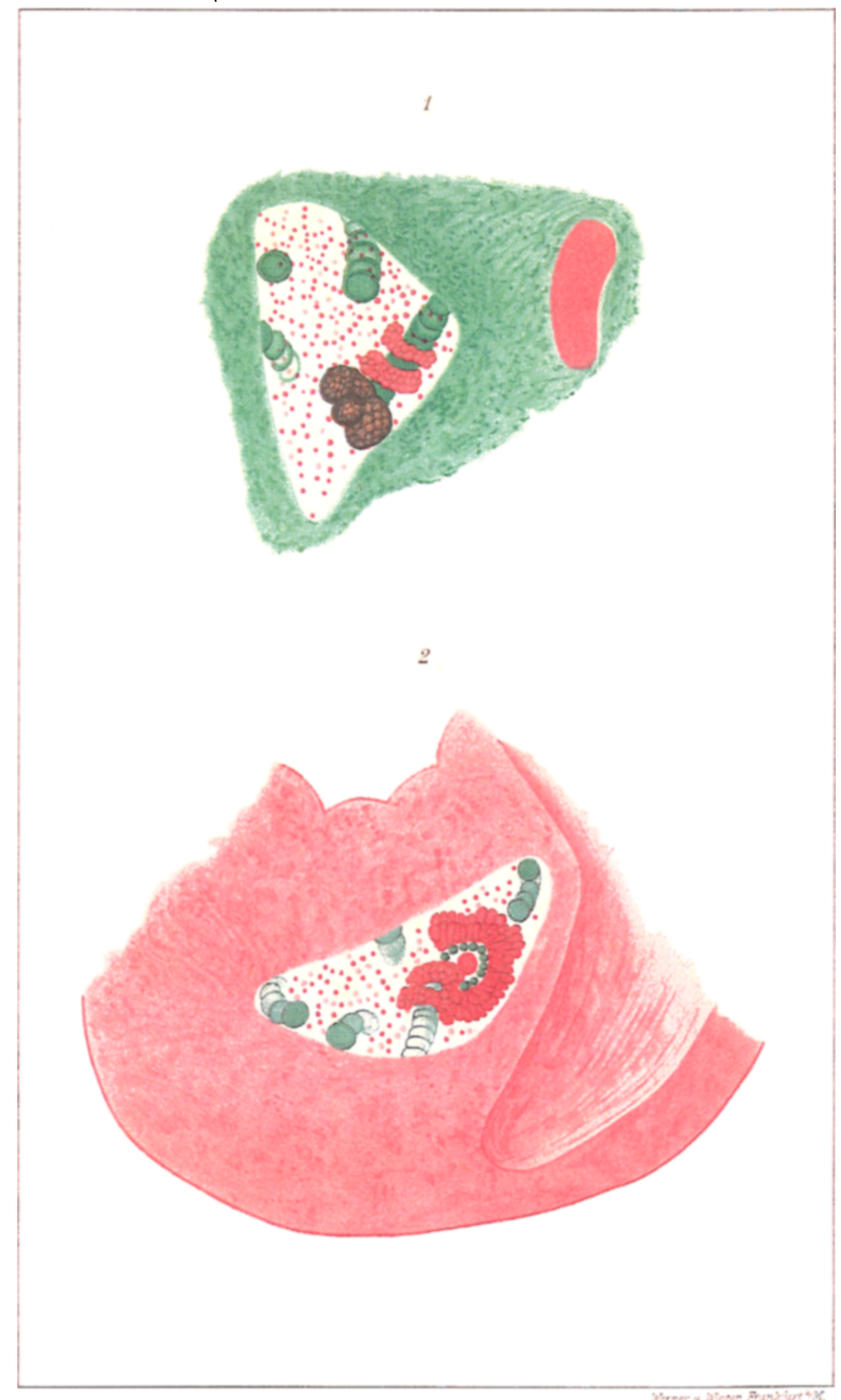

